Materials and Methods The resident validated prescriptions every day, could consult medical files in the Neurology ward and attended medical clinical rounds twice weekly. When a problem was identified in a prescription, the resident discussed it directly with the physician. Every PI was collected using a validated record sheet (Conort et al, J Pharm Clin, 2004).

Results The resident made 95 interventions during the eighteenweek study period. The physician acceptance rate of these recommendations was $92 \%$. The most commonly identified drug-related problems were: inappropriate administration (19\%), non-indicated drug $(17 \%)$ and under dosage (12\%). Nervous system drugs $(24 \%)$, alimentary tract and metabolism drugs $(17 \%)$ and cardiovascular drugs (14\%) were the most frequently involved.

Conclusions The regular presence of the pharmacy resident on the neurology ward enabled him to be well integrated and to become familiar with inpatient specificities in the neurology department. Collaborative working relationships between pharmacists and physicians are the key to success and to reducing the number of potentially inappropriate prescriptions. The high physician acceptance rate is a good indication of intervention relevance. Recurrent problems were identified during this study. Data on interventions were presented to the pharmacy and therapeutic committee.

No conflict of interest.

\section{CPC-005 A STUDY TO EVALUATE USTEKINUMAB IN PATIENTS WITH MODERATE TO SEVERE PLAQUE PSORIASIS}

doi:10.1136/ejhpharm-2013-000276.462

E Marquez Fernandez, E Sanchez Yañez, C Lara Ramos, JM Fernandez Ovies, C Gonzalez-Perez, B Muros De Fuentes. Hospital Clinico Virgen De La Victoria, Pharmacy, Malaga, Spain

Background Ustekinumab is a monoclonal antibody that binds with specificity and affinity to the p40 subunit of cytokines IL-12 and IL-23.

Purpose To determine the short and long-term effectiveness of ustekinumab in patients with moderate to severe plaque psoriasis.

To determine the change from the baseline in the Dermatology Life Quality Index (DLOI).

To describe the safety profile of ustekinumab in clinical practise. Materials and Methods We reviewed the medical records of 31 patients who had been prescribed ustekinumab between October 2009 and July 2012 in our hospital. We noted the PASI (Psoriasis area severity index) and DLOI scores before and during the treatment and the adverse events reported by patients in their cheque-ups.

Clinicians typically consider at least 75\% improvement (PASI75) in the disease to be a clinically meaningful improvement indicative of success.

Results Data were unavailable in 3 patients.

$42.4 \%$ (12) of patients were male and the median age was 44 years. The median baseline PASI score was 17.89 and the mean duration of psoriasis was 23.22 years.

15 patients (54\%) completed a DLOI questionnaire. The median baseline DLOI score was 15.93 and the median DLOI score during the treatment was 1.26 .

7 patients (25\%) reported adverse events:

4 patients (14.4\%) upper respiratory tract infection.

2 patients $(7.2 \%)$ dyslipidaemia.

1 patient $(3.6 \%)$ liver enzyme alteration.

1 patient $(3.6 \%)$ basal-cell carcinoma.

1 patient (3.6\%) generalised desquamative erythema.

There was only one adverse event that forced the suspension of treatment (generalised desquamative erythema).
Conclusions In our study, ustekinumab demonstrated a rapid onset of action and a high effectiveness, stable safety and a great improvement in the quality of life in patients with moderate to severe plaque psoriasis on up to 34 months of continuous therapy.

Abstract CPC-005 Table 1

\begin{tabular}{|c|c|c|c|c|c|c|}
\hline & $\begin{array}{l}16 \text { Weeks } \\
n=28\end{array}$ & $\begin{array}{l}6 \text { Months } \\
n=23\end{array}$ & $\begin{array}{l}12 \text { Months } \\
n=17\end{array}$ & $\begin{array}{l}18 \text { Months } \\
n=14\end{array}$ & $\begin{array}{l}24 \text { Months } \\
n=13\end{array}$ & $\begin{array}{l}30 \text { Months } \\
n=10\end{array}$ \\
\hline PASI75(\%) & 82.1 & 91.3 & 94.1 & 92.9 & 76.9 & 90 \\
\hline PASI90(\%) & 71.4 & 69.6 & 47.1 & 50 & 46.2 & 50 \\
\hline
\end{tabular}

No conflict of interest.

\section{CPC-006 ADEQUACY OF CRITERIA FOR STARTING NATALIZUMAB IN PATIENTS WITH MULTIPLE SCLEROSIS}

doi:10.1136/ejhpharm-2013-000276.463

B Marzal Alfaro, A De Lorenzo Pinto, MS Pernía López, B Cáliz Hernández, Ml Yeste Gómez, P Arrabal Duran, M Sanjurjo Saez. Hospital General Universitario Gregorio Marañon, Pharmacy, Madrid, Spain

Background Natalizumab is a monoclonal antibody authorised as second-line treatment after failure with interferon beta or in rapidly evolving severe relapsing-remitting multiple sclerosis (RRMS). Due to its high cost and safety profile, the appropriate selection of patients who will benefit most is of paramount importance.

Purpose To evaluate the adequacy of criteria for starting treatment with natalizumab in patients with multiple sclerosis (MS) based on the protocol approved in a tertiary hospital.

Materials and Methods Observational, retrospective analysis of patients treated with natalizumab between 2008 and 2011. Study data were obtained from clinical records.

Results 31 patients were treated with natalizumab, 26 women (83.9\%) and 5 men (16.1\%). Mean age was 38.8 years (SD $=9.1$ ). Mean time between diagnosis and natalizumab start was 7.8 years ( $\mathrm{SD}=5.9) .29$ patients (93.5\%) had RRMS, 1 secondary-progressive MS (SPMS) and the other an intermediate disease between RRMS and SPMS. The mean number of relapses before treatment started was $3.7(\mathrm{SD}=1.5)$ and the mean score for the expanded disability status scale was 3.3 (range 1-6). 27 patients (87.1\%) had previously been treated with immunomodulatory drugs (interferon beta). In 4 patients (12.9\%) natalizumab was first line treatment. All were diagnosed with rapidly evolving severe RRMS with gadoliniumenhancing lesions in brain magnetic resonance imaging and more than 2 disabling relapses in the previous year. At the end of the study 22 patients continued treatment and 9 had finished. These latter patients were categorised in two groups: short treatment duration (4 patients, median 5 months) and long treatment duration (5 patients, median 24 months).

Conclusions In our population, adequacy of criteria for starting treatment with natalizumab is appropriate and the drug was used for the authorised indications in more than $90 \%$ of patients.

No conflict of interest.

\section{CPC-007 ADHERENCE PROBLEMS IDENTIFIED BY MOTIVATIONAL INTERVIEWING AND MEDICINES REVIEW IN STROKE PATIENTS}

doi:10.1136/ejhpharm-2013-000276.464

'U Hedegaard, ${ }^{2} \mathrm{LJ}$ Kjeldsen, ${ }^{3} \mathrm{~J}$ Hallas. 'University of Southern Denmark, Institute of Public Health Clinical Pharmacolgy, Odense, Denmark; ${ }^{2}$ Amgros I/S, The Danish Research Unit for Hospital Pharmacy, Copenhagen, Denmark; ${ }^{3}$ University of Southern Denmark, Institute of Public Health Clinical Pharmacology, Odense, Denmark

Background Poor adherence to secondary prevention medicines occurs frequently in patients suffering a stroke or Transient 